\title{
Stuart C. Mendel and Jeffrey L. Brudney: Partnerships the Nonprofit Way: What Matters, What Doesn't
}

\section{Book Review}

\author{
${ }^{1}$ Marxe School of Public and International Affairs, Baruch College, City University of New York, New York, USA, E-mail: \\ john.casey@baruch.cuny.edu
}

DOI: $10.1515 / \mathrm{npf}-2018-0030$

Stuart C.Mendel and Jeffrey L.Brudney (2018). Partnerships the Nonprofit Way: What Matters, What Doesn't. Bloomington, IN; Indiana University Press. 188 pages.

Stuart Mendel and Jeffrey Brudney offer an excellent overview of the current state of scholarship and practice regarding the partnerships that nonprofits establish with governments, with for-profit businesses, and with other nonprofits. As the authors note, the overall goal of these partnerships is to generate higher efficiencies, strengthen service delivery capacity and increase public value in order to amplify the reach of public serving programs. In 183 pages of text and endnotes, Mendel and Brudney draw on their extensive survey work with nonprofit executives to identify the partnership principles that ensure that the work of nonprofits is effective, positive, durable and replicable. The book covers the three possible dyadic pairs for partnerships involving the three sectors: government-nonprofits; business-nonprofits; nonprofits-nonprofits.

Most significantly for those of us writing about and working in this sector, the book takes an unashamedly "nonprofit-first" approach, which focuses on the dynamics of partnerships from the nonprofit perspective. Nonprofits are the central actors in this research and the authors emphasize that only by prioritizing the nonprofits' mission and culture will such partnerships be successful. The focus in particular is on the concept of the "three missions"; those of the two partners and the key third mission of the partnership itself.

The book also examines partnerships by subsectors. One chapter highlights the peculiarities of partnerships in human and social service, public and societal benefits, health, arts and culture, and education. Another chapter is dedicated to analyzing grant makers' partnership practices.

The specter of a possible distinction between collaboration and partnership appears throughout the book. In the first chapter, Mendel and Brudney talk of the "subtle distinctions of collaboration and partnership drivers" and that "partnership is more characterized by the heightened role of key executives" (p. 2), but by the end of the book the difference between the terms is never quite resolved. A good demonstration of the continuing confusion appears, perhaps inadvertently, in an endorsement on the back cover. A reviewer notes that "[the book] shines a ... light on the factors that allow nonprofits and their partners to succeed in their collaborative endeavors." So is the book about partners in collaborative endeavors, about collaborations in partnerships, or about how to move from collaboration to partnership? Does the distinction matter? Perhaps not, as the book delivers important insights on how to build strong partnerships - and collaborations - and how to measure their continued success. However, the book could have benefited from a more direct analysis of this issue, and more explicit discussion of the use of the terms.

To quote the subtitle of the book, What Matters and What Doesn't? The book provides ample details about what matters. The characteristics of good partnerships are identified and in the final chapters there are extensive listings of benchmarks and other indicators

While I endorse the nonprofit-first approach in this book, I did miss some more cross-referencing with analyses of partnership literature from other sectors, in particular the considerable academic and professional literature about business-business partnerships and about business-nonprofit partnerships written from the perspective of business. As the authors note, partnerships in the three dyads have different logics, as do partnerships in the different subsectors, and the book does a good job in identifying and analyzing them, but I kept wondering exactly how the analyses, concepts, and theorizing from the nonprofit-first perspective might be different from those from the lenses of the other two sectors. 
All partnerships have their peculiarities but, as the final chapters demonstrate, in the end they are subject to an identifiable suite of principles and measured by similar indicators. Key concepts such as trust and reciprocity are essential elements of all partnerships between organizations, and between the people in them. It is emblematic - and I assume not just a coincidence - that Chapter 4 is titled Good to Great, the same title as the mega-selling business book by Jim Collins. Collins published an addendum Good to Great and the Social Sectors: Why Business Thinking is Not the Answer which specifically contrasted the sectors, and this book would have benefited from some of the same analysis. The Mendel and Brudney book is sprinkled with concepts and processes that first emerged in the business sector - e. g. transformational versus transactional management, return-on-investment (ROI), dashboards - and some more acknowledgement of these crossovers was perhaps needed.

The book is about building grounded theory that goes beyond experience and anecdotes. In the Acknowledgment the authors state that their intention was to go beyond derivative theory - theory drawn from theory (p. vii). Implicit then is that the also wanted to go beyond derivative practice - practice drawn from practice and on page 165 they distinguish between theories of partnership and real-world indicators. But, in the end, the theory they establish reads a lot like the pointers you might find in a best practice manual. When analyzing real-world processes in organizations, theory and practice are often not that dissimilar. My first impression on seeing many of the summary figures and tables, is that they are not only theorizing, but in effect essential advice for anyone working to improve partnerships or seeking to understand why some succeed and others fail.

In the final chapter, Towards Nonprofit Theory, Table 7.1, the summary table of the section Component Theories of Nonprofit Partnerships would not be out of place in a practice manual published by an industry association or consultant firm, and the chapter (and the book itself) finishes with a section on dashboards, a quintessential management tool of the modern executive in all three sectors. So can we claim that we can distinguish theory from practice when researching real-world organizational dynamics? If the basis of theory is that the gathering, analysis and systemizing of data leads to more structured insights, then this book succeeds masterfully. If the objective is to take the reader beyond where practice manuals could ever hope to go, it may not have fully achieved that.

I have one gripe about the book format. Unfortunately, it uses only endnotes for each chapter, but has no final bibliography. A reader may want to find if there are references to a specific author or if an author has more than one publication, but in this format it's not possible. (I stumbled across a reference to my own work buried in an endnote). The book would have also have benefited from a more consistent chapter structure. The default structure seem to be to start with an Overview, then give an Introduction, and to end with a Conclusion. But some chapters also have a final In Review section that would have been good to include in every chapter.

Despite any problems, what matters is that the book is an important contribution to the literature on the partnership work of nonprofits. The stated audience of the book includes scholars, students, nonprofit executives, volunteers and grant makers. All of them could gain important insights into partnerships by reading this book. The core lesson from the book and, more importantly, from the nonprofit executives who were interviewed as part of the research, appears to be a version of trust and verify. Mendel and Brudney clearly lay out why partnerships are important and give clear instructions on how to achieve success and measure outcomes. 